\title{
NULL SPACES DIMENSION OF THE EIGENVALUE -1 IN A GRAPH
}

\author{
Gohdar H. Mohiaddin ${ }^{a}$, Khidir R. Sharaf ${ }^{\text {a }}$ \\ ${ }^{a}$ Dept. of Mathematic, Faculty of Science, University of Zakho, Kurdistan Region, Iraq - (gohdar.mohiaddin, \\ khidir.sharaf)@uoz.edu.krd
}

Received: Jul., 2019/ Accepted: Oct., 2019/Published: Dec.,2019

https://doi.org/10.25271/sjuoz.2019.7.4.609

\begin{abstract}
:
In geographic, the eigenvalues and eigenvectors of transportation network provides many informations about its connectedness. It is proven that the more highly connected in a transportation network $\mathrm{G}$ has largest eigenvalue and hence more multiple occurrences of the eigenvalue -1 . For a graph $\mathrm{G}$ with adjacency matrix $\mathrm{A}$, the multiplicity of the eigenvalue -1 equals the dimension of the null space of the matrix A + I.

In this paper, we constructed a high closed zero sum weighting of $\mathrm{G}$ and by which its proved that, the dimension of the null space of the eigenvalue -1 is the same as the number of independent variables used in a non-trivial high closed zero sum weighting of the graph. Multiplicity of -1 as an eigenvalue of known graphs and of corona product of certain classes of graphs are determined and two classes of -1- nut graphs are constructed.
\end{abstract}

KEYWORDS: Graph Theory, High Zero Sum Weighting, Adjacency Matrix, Nullity, Corona Product.

\section{INTRODUCTION}

A simple graph $\mathrm{G}$ with vertex set $\mathrm{V}, \mathrm{V}=\mathrm{v}_{1}, \mathrm{v}_{2}, \ldots, \mathrm{v}_{\mathrm{n}}$, is singular provided that its adjacency matrix $A(G)$ is a singular matrix. The eigenvalues $\lambda_{1}, \lambda_{2}, \ldots, \lambda_{n}$ of $\mathrm{A}(\mathrm{G})$ are said to be the eigenvalues of the graph $\mathrm{G}$, which form the spectrum of $\mathrm{G}$, denoted by $\mathrm{Sp}(\mathrm{G})$. The occurrence of zero as an eigenvalue in the spectrum of the graph $\mathrm{G}$ is called its nullity and is denoted by $\eta$ or $\eta(G)$. The nullity $\eta(\mathrm{G})$ is the dimension of the null space of $A(G)$ (Cheng and Liu, 2007). The nullity $\eta(\mathrm{G})=\mathrm{n}$ if, and only if, $\mathrm{G}$ is the empty (null) graph. Let $\mathrm{G}=\mathrm{G}_{1} \cup \mathrm{G}_{2} \cup \mathrm{G}_{3} \cup, \ldots, \mathrm{UG}_{\mathrm{k}}$ then, $\eta(\mathrm{G})=\sum_{i=1}^{k} G_{i}$ where $\mathrm{G}_{1}, \mathrm{G}_{2}, \quad \ldots \quad, \mathrm{G}_{\mathrm{k}}$ are connected components of G See (Ali, et al., 2016a; Ali et al., 2019).

A vertex weighting of a graph $G$ is a function $f: V(G) \rightarrow \mathbb{R}$ where $\mathbb{R}$ is the set of real numbers, which assigns real numbers (weights) to each vertex. The weighting of $G$ is said to be non-trivial if there is at least one vertex $v \in V(G)$ for which $\mathrm{f}(\mathrm{v}) \neq 0$. A non-trivial vertex weighting of a graph $\mathrm{G}$ is called a zero-sum weighting provided that for each $\mathrm{v} \in \mathrm{V}$ (G), $\sum f(u)=0$, where the summation is taken over all $\mathrm{u} \in$ $\mathrm{N}(\mathrm{v})$. Out of all zero-sum weightings of a graph G, a high zero-sum weighting (hzsw) of $\mathrm{G}$ is one that uses a maximum number of non-zero independent variables (Sharaf and Rashed, 2002).

A graph $\mathrm{G}$ is a Smith graph if 2 is an eigenvalue of G, a 2weighting technique is applied to characterize some classes of Smith graphs as well as to study the nullity of vertex identification of such graphs. It is also proved that under certain conditions the vertex identification of some Smith graphs is a Smith graph (Mohiaddin and Khidir, 2018).

In other words a graph with nullity $\eta$ contains cores determined by a basis for the null space of A. A singular graph, on at least two vertices, with a kernel eigenvector having no zero entries, is said to be a core graph.

The removal of a vertex $v$ from a graph $G$ results in a subgraph $G-v$ of $G$ consisting of all vertices of $G$ except $v$, and all edges not incident with v. For two distinct vertices a and $b$ of a graph $G$, we define $G+a b$ to be a graph obtained from $\mathrm{G}$ by adding a new vertex ab which is adjacent to all vertices in $N(a) \cup N(b)$, the graph $G . a b$ is $G+a b-a-b$.

Definition 1.1 (Mohan et al., 2017). Let $\mathrm{G}_{1}$ and $\mathrm{G}_{2}$ be two graphs with vertex set $\mathrm{V}\left(\mathrm{G}_{1}\right)=\left\{\mathrm{v}_{1}, \mathrm{v}_{2}, \ldots, \mathrm{v}_{\mathrm{n} 1}\right\}$ and $\mathrm{V}\left(\mathrm{G}_{2}\right)=\left\{\mathrm{u}_{1}, \mathrm{u}_{2}, \ldots\right.$ , $\left.\mathrm{u}_{\mathrm{n} 2}\right\}$ respectively, the corona of $\mathrm{G}_{1}$ and $\mathrm{G}_{2}$ denoted by $\mathrm{G}_{1} \mathrm{O} \mathrm{G}_{2}$ is defined as taking one copy of $\mathrm{G}_{1}$ and $\mathrm{n}_{1}$ copies of $\mathrm{G}_{2}$ by adjoining $i^{\text {th }}$ vertex of $G_{1}$ to each vertex of $G_{2}$ in $i^{\text {th }}$ copy.

Many invariant of the corona product $\mathrm{G}_{1} \mathrm{O} \mathrm{G}_{2}$ such as nullity, domination number, colorability are related to the invariants of $\mathrm{G}_{1}$ and of $\mathrm{G}_{2}$.

\section{Theorem 1.2 (Interlacing Theorem)}

(Brouwer and Haemers, 2011). Let G be a graph with eigenvalues $\lambda_{1} \geq \lambda_{2} \geq \ldots \geq \lambda_{n}$, and let the eigenvalues of $G-v$ be $\mu_{1} \geq \mu_{2} \geq$ $\ldots \geq \mu_{n-1}$. Then the eigenvalues of $G-v$ are interlaced with the eigenvalues of $G$, that is, $\lambda_{1} \geq \mu_{1} \geq \lambda_{2} \geq \mu_{2} \geq \ldots \geq \mu_{n-1} \geq \lambda_{n}$.

A vertex of a graph can be a core vertex if, on deleting the vertex, the nullity decreases, or a Fiedler vertex, otherwise. They adopt a graph theoretical approach to determine conditions required for the identification of a pair of prescribed types of root vertices of two graphs to form a cut-vertex of unique type in the coalescence. Moreover, the nullity of subgraphs obtained by perturbations of the coalescence graph is determined relative to its nullity (Ali et al., 2016a).

The change in nullity when graphs with a cut-edge, and others derived from them, undergo geometrical operations, the deletion of edges and vertices, the contraction of edges and the insertion of an edge at a coalescence vertex are studied in (Ali et al., $2016 \mathrm{~b}$ ). It is proved that, two connected labeled graphs $\mathrm{H}_{1}$ and $\mathrm{H}_{2}$ of nullity one, with identical one-vertex deleted subgraphs $\mathrm{H}_{1}$ $-\mathrm{z}_{1}$ and $\mathrm{H}_{2}-\mathrm{Z}_{2}$ and having a common eigenvector in the nullspace of their $0-1$ adjacency matrix, can be overlaid to produce the superimposition graph $\mathrm{G}$. The graph $\mathrm{G}$ is $\mathrm{H}_{1}+\mathrm{z}_{2}$ and also $\mathrm{H}_{2}+\mathrm{Z}_{1}$ whereas $\mathrm{G}+\mathrm{e}$ is obtained from $\mathrm{G}$ by adding the edge $\mathrm{z}_{1} \mathrm{Z}_{2}$ (Ali et al., 2019).

* Corresponding author

This is an open access under a CC BY-NC-SA 4.0 license (https://creativecommons.org/licenses/by-nc-sa/4.0/) 
In this paper the dimension of the null space of the eigenvalue -1 of a simple graph by introducing a new idea, denoted by high closed zero sum weighting is studied.

Finally, $K_{n}, K_{a, b}, C_{n}, P_{n}$ are complete, complete bipartite, cycle and path graphs of order $n$, for more definition and details see (Cvetkovic et al., 1979). All graphs mentioned in the following are connected graphs except where stated.

\section{MULTIPLICITY OF THE EIGENVALUE -1 IN A GRAPH}

In this section, we define the occurrence of -1 as an eigenvalue of a graph $\mathrm{G}$. It is clear that -1 is an eigenvalue of a matrix $A$ if and only if determinant of the matrix $A+I$ is equal to zero.

Definition 2.1 A non-trivial vertex weighting of a graph $G$ is called a closed zero-sum weighting provided that for each $v \in V(G)$, then $\sum f(u)=0$, where the summation is taken over all $u \in N[v]$. Out of all closed zero-sum weightings of a graph $\mathrm{G}$, a high closed zero-sum weighting (hczsw) of $\mathrm{G}$ is one that uses maximum number of non-zero independent variables.

Proposition 2.2 For any graph $G,-1$ is an eigenvalue of $G$ iff $G$ possesses a closed zero-sum weighting.

Proof. Let A be the adjacency matrix of G. If -1 is an eigenvalue of $\mathrm{G}$ then, the matrix $\mathrm{A}+\mathrm{I}$ is a singular matrix and hence there exist a non-zero vector $\mathrm{X}$ such that $(\mathrm{A}+\mathrm{I}) \mathrm{X}=0$. This, means that $G$ possesses a closed zero-sum weighting. On the other hand, if $G$ possesses a closed zero-sum weighting,

then, $(A+I) X=0$, where $X$ is a non zero vector. Hence $A X=$ $-\mathrm{X}$ which proves that -1 is an eigenvalue of $\mathrm{G}$.

It is easy to show that, for the next largest eigegvalue $\lambda_{2}(\mathrm{G})$ to be -1 the graph must be complete and for the third largest eigenvalue $\lambda_{3}(G)$ it is known that $\lambda_{3}(G)=-1$ iff $G^{\mathrm{c}}$ is isomorphic to the union of a complete bipartite graph and some isolated vertices.

Note : We denote the multiplicity of -1 as an eigenvalue of a graph $\mathrm{G}$ by $\mathrm{m}_{-1}(\mathrm{G})$.

Here we give some illustrated graphs with hczsws, which classify that $\mathrm{m}_{-1}\left(\mathrm{C}_{3}\right)=2$ and $\mathrm{m}_{-1}\left(\mathrm{P}_{6}+\mathrm{e}\right)=2$, where $\mathrm{e}=\mathrm{v}_{4} \mathrm{v}_{6}$ in the usual labeling of the path $\mathrm{P}_{6}$.
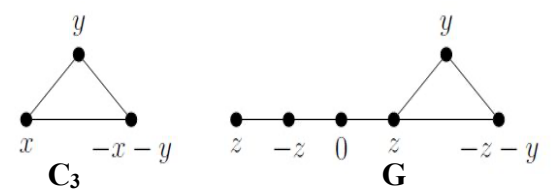

Figure 1. hezsw for $\mathrm{C}_{3}$ and $\mathrm{G}$.

Theorem 2.3 In a graph $G, m_{-1}(G)$ equals the maximum number of independent variables in a hczsw of $G$ and conversely.

Proof. Let A be the adjacency matrix of G, and I be the identity matrix. -1 is an eigenvalue of $\mathrm{G}$ with multiplicity $\mathrm{m}$, if and only if zero is an eigenvalue of the matrix A + I with the same multiplicity $\mathrm{m}$. There exist a hzsw of $\mathrm{G}$ that uses exactly $\mathrm{m}$ independent variables, Say $\mathrm{X}$. Thus $(\mathrm{A}+\mathrm{I}) \mathrm{X}=0$ gives $\mathrm{AX}=-\mathrm{IX}$, then the same number of independent variables is used in a hczsw for G.

Corollary 2.4 The following are equivalent :

i) -1 is an eigenvalue of a graph $G$.

ii) The matrix $A+I$ is a singular matrix.

iii) There exist a non zero eigenvector $X$ such that $A X=-X$. iv) G possesses a closed zero-sum weighting.

Proof. Is direct.

Theorem 2.5 If $G$ is $r$-regular graph and $r=\lambda_{I} \geq \lambda_{2} \geq \ldots \geq \lambda_{n}$ are the eigenvalues of $G$, then the eigenvalues of $G^{c}$ are $n-1-r$ and $\left\{-1-\lambda_{i} ; 2 \leq i \leq n\right\}$.

Proof. Because $\mathrm{A}\left(\mathrm{G}^{\mathrm{c}}\right)=\mathrm{J}-\mathrm{I}-\mathrm{A}(\mathrm{G})$, where $\mathrm{J}$ is an $\mathrm{n} \times \mathrm{n}$ matrix in which each entry is one and $I$ is the identity matrix; $G$ is (n-1-r)regular, so the largest eigenvalue is $n-1-r$ with corresponding eigenvalue $\mathrm{Y}=[1,1, \ldots, 1]^{\mathrm{t}}$.

Any other eigenvalue $\lambda$ has an eigenvector $\mathrm{X}$ orthogonal to $\mathrm{Y}$, and hence $A\left(G^{c}\right) X=(J I-A(G)) X=(0-1-\lambda) X$.

Corollary 2.6 For any graph $G$ having at least 2 vertices $\left|\mathrm{n}(G)-m_{-1}\left(G^{c}\right)\right| \leq 1$ strictly holds if $G=P_{4 n+3}$. Equality holds if $G=K_{n}$.

Proof. Direct from Theorem 2.3.

\section{MULTIPLICITY OF - 1 AS AN EIGENVALUE IN SOME KNOWN GRAPHS}

Let $\mathrm{H}$ be any graph in which the multiplicity of -1 is $\mathrm{m}$, then we prove the following:

Lemma 3.1 If $G$ is a graph obtained by identifying an end vertex of $P_{4}$ with any vertex of a graph $H$ then, $m_{-1}(G)=m_{-1}(H)$.

Proof. Label the vertices of $\mathrm{P}_{4}$ in a usual way $\mathrm{v}_{1}, \mathrm{v}_{2}, \mathrm{v}_{3}$ and $\mathrm{v}_{4}$ where $\mathrm{v}_{4}$ is the identified vertex with a vertex of the graph $\mathrm{H}$. In a hczsw of $G$ there are two possibilities for the weighting of the identified vertex $\mathrm{v}_{4} \equiv \mathrm{v}$.

a) If $w(v)=0$ then, $w\left(v_{1}\right)=w\left(v_{2}\right)=w\left(v_{3}\right)=0$ and hence removing $\mathrm{v}_{1} ; \mathrm{v}_{2}$ and $\mathrm{v}_{3}$ will not change any weight in the hczsw of $\mathrm{H}$ so $\mathrm{m}$. ${ }_{1}(\mathrm{G})=\mathrm{m}_{-1}(\mathrm{H})$.

b) If $w(v) \neq 0$ then, $w\left(v_{1}\right)=x, w\left(v_{2}\right)=-x$ and $w\left(v_{3}\right)=0$ and again removing $v_{1}, v_{2}$ and $v_{3}$ will also not change any weight in the hczsw of $H$ so, $m_{-1}(G)=m_{-1}(H)$. See Figure 2 .

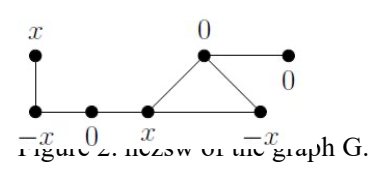

Proposition 3.2 Null spaces dimension of -1 as an eigenvalue of a complete graph $K_{n}$ is defined as:

$$
m_{-1}\left(K_{n}\right)=\left\{\begin{array}{lll}
0 & \text { if } & n=1 \\
n-1 & \text { if } & n \neq 1
\end{array}\right.
$$

Proof. Is direct, from the number of distinct variables in a hczsw of $\mathrm{K}_{\mathrm{n}}$, namely $\mathrm{x}_{1}, \mathrm{x}_{2}, \ldots, \mathrm{x}_{\mathrm{n}-1},-\mathrm{x}_{1},-\mathrm{x}_{2}, \ldots-\mathrm{x}_{\mathrm{n}-1}$.

Proposition 3.3 Null spaces dimension of -1 as an eigenvalue of a path $P_{n}$ is defined as:

$$
m_{-1}\left(P_{n}\right)=\left\{\begin{array}{l}
1 \quad \text { if } \quad n=2 \bmod 3 \\
0 \quad \text { otherwise }
\end{array}\right.
$$

Proof. Assume that $\mathrm{n}=2$, then a hczsw of $\mathrm{P} 2$ is $\mathrm{x}$, $-\mathrm{x}$ and hence 1 is an eigenvalue of $P_{2}$ with multiplicity 1 . For $n=2 \bmod 3$, any hczsw of $P_{n}$ is of the form $x,-x, 0, x,-x, \ldots, 0, x,-x$ where the triple $0, x,-x$ is repeated $(n-2) / 3$ times. Hence, dimension of the null space of -1 is 1 . If $n \neq 2 \bmod 3$, then there exist no non trivial hczsw for $\mathrm{P}_{\mathrm{n}}$. That is, the dimension of the null space of -1 is zero. $\square$

Proposition 3.4 Null spaces dimension of -1 as an eigenvalue of a cycle $C_{n}$ is defined as:

$$
m_{-1}\left(C_{n}\right)= \begin{cases}2 & \text { if } \quad n=3 \\ 1 & \text { if } n=0 \bmod 3 \text { and } n \neq 3 \\ 0 & \text { otherwise }\end{cases}
$$

Proof. Assume that $\mathrm{n}=3$, then a hezsw of $\mathrm{C}_{3}$ is $\mathrm{x}, \mathrm{y},-\mathrm{x}-\mathrm{y}$ which uses 2 independent variables, hence -1 is an eigenvalue of $C_{3}$ with multiplicity 2 . 
For $\mathrm{n}=0 \bmod 3$, any hczsw of $\mathrm{C}_{\mathrm{n}}$ is of the form $\mathrm{x},-\mathrm{x}, 0, \mathrm{x},-$ $\mathrm{x}, \ldots, 0, \mathrm{x},-\mathrm{x}, 0$ where the triple $\mathrm{x},-\mathrm{x}, 0$, is repeated $\mathrm{n} / 3$ times. Thus, dimension of the null space of -1 is 1 . If $n \neq 0$ mod 3 then, there exist no non trivial hczsw for $\mathrm{C}_{\mathrm{n}}$, that is, the dimension of the null space of -1 is zero.
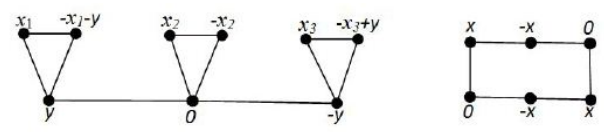

Figure 3. A hczsw for a graph with null space of dimension 4 and a hczsw of $\mathrm{C}_{6}$.

For the complete graph $\mathrm{K}_{\mathrm{n}}$, there exist only one hczsw that uses exactly $n-1$ independent variables namely, $x_{1}, x_{2}, x_{3}, \ldots$, $\mathrm{x}_{\mathrm{n}-1},-\mathrm{x}_{1}-\mathrm{x}_{2}-\mathrm{x}_{3}-\ldots-\mathrm{x}_{\mathrm{n}-1}$, this proves that the dimension of the null space of -1 of the complete graph $K_{n}$ is $n-1$. By Interlacing Theorem (adding or removing a vertex to a graph changes the dimension of the null space of any eigenvalue by at most one) So, if $t$ isolated vertices are adjacent to distinct vertices of a complete graph $\mathrm{K}_{\mathrm{n}}$, where $\mathrm{t} \leq \mathrm{n}-1$, to form a graph $K_{n}^{t}$. Then

$\mathrm{m}_{-1}\left(K_{n}^{t}\right)==\mathrm{n}-1-\mathrm{t}$. See Figure 3 where $\mathrm{n}=4$ and $\mathrm{t}=2$.

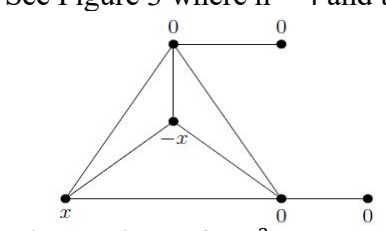

Figure 4. hezsw for $K_{4}^{2}$.

Proposition 3.5 The dimension of the nullspace of -1 as an eigenvalue of a complete bipartite graph $\mathrm{K}_{\mathrm{a}, \mathrm{b}}$ is:

$$
m_{-1}\left(\mathrm{~K}_{\mathrm{a}, \mathrm{b}}\right)= \begin{cases}1 & \text { if } \quad \begin{array}{c}
a=b=1 \\
0
\end{array}\end{cases}
$$

Proof. Any hczsw of $\mathrm{K}_{\mathrm{a}, \mathrm{b}}$ must satisfy: $\mathrm{x}_{\mathrm{i}}+\sum y_{j}=0$ and $\mathrm{y}_{\mathrm{j}}+$ $\sum x_{i}=0$, for $\mathrm{i}=1,2, \ldots$, a and $\mathrm{j}=1,2, \ldots, \mathrm{b}$ which gives $\mathrm{x}_{1}$ $=\mathrm{x}_{2}=\ldots=\mathrm{x}_{\mathrm{a}}$ and $\mathrm{y}_{1}=\mathrm{y}_{2}=\ldots=\mathrm{y}_{\mathrm{b}}$. Then $\mathrm{x}_{1}=-\mathrm{ay}_{1}$ and $\mathrm{y}_{1}=$ $-\mathrm{bx}_{1}$, thus $\mathrm{x}_{1}=\mathrm{bax}_{1}$, hence $\mathrm{ba}=1$ that is $\mathrm{a}=\mathrm{b}=1$. So, the dimension of the nullspace of -1 as an eigenvalue of a complete bipartite graph $\mathrm{K}_{1,1}$ is one and zero otherwise. 口

Existence of a triangle in a graph $\mathrm{G}$ is not a sufficient condition to have -1 as an eigenvalue, but if the graph $\mathrm{G}$ is of the form $\mathrm{C}_{3} \bullet \mathrm{H}$, then -1 is an eigenvalue because of the czsw say $\mathrm{x},-\mathrm{x}, \quad 0$ of vertices of $\mathrm{C}_{3}$ where weight of the identified vertex is 0 . This gives the prove of the next corollary.

Corollary 3.6 For the graph $\mathrm{C}_{3} \bullet \mathrm{H}$ we have: $m_{-1}\left(\mathrm{C}_{3} \bullet \mathrm{H}\right) \leq m_{-1}(\mathrm{H})+1$

Now, given any number $\mathrm{n}$ it is easy to construct a tree $\mathrm{T}$ in which the dimension of the nullspace of -1 of $T$ is $n$ by using hczsws technique. To do this insert a new vertex to each edge of the star graph $S_{1,2 n}$, then the hczsw of this tree is $0 ; x 1$,$\mathrm{x} 1 ;-\mathrm{x}_{1} ; \mathrm{x}_{1}, \mathrm{x}_{2},-\mathrm{x}_{2},-\mathrm{x}_{2}, \mathrm{x}_{2}, \ldots, \mathrm{x}_{\mathrm{n}},-\mathrm{x}_{\mathrm{n}},-\mathrm{x}_{\mathrm{n}}, \mathrm{x}_{\mathrm{n}}$, where 0 is the weight of the central vertex and other vertices, are alternatively positive and negative for each variable.

\section{VERTEX IDENTIFICATION}

The following results will be proved for a vertex identi_cation of two graphs $G_{1}$ and $G_{2}$ with rooted vertices $v_{1}$ and $v_{2}$.

Definition 4.1 In a czsw of a graph $G$ each vertex with non zero weight is called a -1 -core vertex, and the vector whose entries are weights of such czsw is called a -1-core vector of G.

Definition 4.2 In a hczsw of a graph $G$ each vertex with zero weight is called a -1-core forbidden vertex.

So all vertices of a graph are either -1 -core or -1 - core forbidden vertices and these two types of vertices plays the main rule on the null spaces dimension of the eigenvalue -1 in the vertex identification graph $\mathrm{G}_{1} \bullet \mathrm{G}_{2}$ of two graphs $\mathrm{G}_{1}$ and $\mathrm{G}_{2}$.

Theorem 4.3 For any two graphs $G_{1}$ and $G_{2}$ with rooted vertices $v_{1}$ and $v_{2}$ we have:

$m_{-1}\left(G_{1}\right)+m_{-1}\left(G_{2}\right)-2 \leq m_{-1}\left(G_{1} \bullet G_{2}\right) \leq m_{-1}\left(G_{1}\right)+m_{-1}\left(G_{2}\right)+1$

Equality holds in the left where $G_{1}=G_{2}=$ triangle and on the right where $G_{1}=G_{2}=$ triangle with a pendant.

Proof. Let $\mathrm{G}_{1}$ and $\mathrm{G}_{2}$ be two graphs with rooted vertices $\mathrm{v}_{1}$ and $\mathrm{v}_{2}$ with hczsws $\mathrm{X}$ and $\mathrm{Y}$. With out loss of generality, assume that $\mathrm{w}\left(\mathrm{v}_{1}\right)=\mathrm{x}_{1}$ and $\mathrm{w}\left(\mathrm{v}_{2}\right)=\mathrm{y}_{1}$ in the above hczsws. Let $\mathrm{z}$ be a hczsw of $G_{1} \bullet G_{2}$ and $\mathrm{z}_{1}$ be the weight of the identified vertex $\mathrm{v}\left(\mathrm{v}_{1} \equiv \mathrm{v}_{2}\right)$. So, we have the following cases:

1) If $\mathrm{x}_{1}=\mathrm{y}_{1}=\mathrm{z}_{1}=0$ then $\mathrm{m}_{-1}\left(\mathrm{G}_{1} \bullet \mathrm{G}_{2}\right)=\mathrm{m}_{-1}\left(\mathrm{G}_{1}\right)+\mathrm{m}_{-1}(\mathrm{G} 2)$ or $\mathrm{m}_{-1}\left(\mathrm{G}_{1} \bullet \mathrm{G}_{2}\right)=\mathrm{m}_{-1}\left(\mathrm{G}_{1}\right)+\mathrm{m}_{-1}\left(\mathrm{G}_{2}\right)+1$.

2) If $\mathrm{x}_{1}=\mathrm{y}_{1}=0$ and $\mathrm{z}_{1} \neq 0$ then $\mathrm{m}_{-1}\left(\mathrm{G}_{1} \bullet \mathrm{G}_{2}\right)=\mathrm{m}_{-1}(\mathrm{G} 1)+\mathrm{m}_{-1}$ $(\mathrm{G} 2)+1$, because a new variable is introduced.

3) If $\mathrm{x}_{1} \neq 0$ and $\mathrm{y}_{1}=0$ or conversely and $\mathrm{z}_{1}=0$ then $\mathrm{m}_{-1}\left(\mathrm{G}_{1} \bullet \mathrm{G}_{2}\right)$ $=\mathrm{m}_{-1}\left(\mathrm{G}_{1}\right)+\mathrm{m}_{-1}\left(\mathrm{G}_{2}\right)-1$, because a variable is vanished.

4) If $\mathrm{x}_{1} \neq 0$ and $\mathrm{y}_{1}=0$ or conversely and $\mathrm{z}_{1} \neq 0$ then $\mathrm{m}_{-1}\left(\mathrm{G}_{1} \bullet \mathrm{G}_{2}\right)$ $=\mathrm{m}_{-1}\left(\mathrm{G}_{1}\right)+\mathrm{m}_{-1}\left(\mathrm{G}_{2}\right)$.

5) If $\mathrm{x}_{1} \neq 0$ and $\mathrm{y}_{1} \neq 0$ and $\mathrm{z}_{1}=0$ then $\mathrm{m}_{-1}\left(\mathrm{G}_{1} \bullet \mathrm{G}_{2}\right)=\mathrm{m}_{-1}\left(\mathrm{G}_{1}\right)$ $+\mathrm{m}_{-1}\left(\mathrm{G}_{2}\right)-2$, because 2 variables are vanished.

6) If $\mathrm{x}_{1} \neq 0$ and $\mathrm{y}_{1} \neq 0$ and $\mathrm{z}_{1} \neq 0$ then $\mathrm{m}_{-1}\left(\mathrm{G}_{1} \bullet \mathrm{G}_{2}\right)=\mathrm{m}_{-1}\left(\mathrm{G}_{1}\right)$ $+\mathrm{m}_{-1}\left(\mathrm{G}_{2}\right)-1$, because 2 variables are replaced by one. But this case does not exist because of the hczsws of the new neighbors of the vertex identified and hczsws of olds one. To get such contradiction, assume that such $\mathrm{z}$ exist, then $\mathrm{z}=\mathrm{ax}=$ by for some $\mathrm{a}$ and $\mathrm{b}$ where, $\mathrm{x}=\mathrm{w}\left(\mathrm{v}_{1}\right)$ in $\mathrm{G}_{1}$ and $\mathrm{y}=\mathrm{w}\left(\mathrm{v}_{2}\right)$ in $\mathrm{G}_{2}$. Then $\sum w\left(N\left(v_{1} \equiv v_{2}\right)\right)$ in $\mathrm{G}_{1} \bullet \mathrm{G}_{2}$ must equal to zero that is ax + $\sum_{G_{1}} w\left(N\left(v_{1}\right)\right)+$ by $+\sum_{G_{2}} w\left(N\left(v_{2}\right)\right)=0$ which implies that $\mathrm{x}+$ $\sum_{G_{1}} w\left(N\left(v_{1}\right)\right)+(1-\mathrm{a}) \mathrm{x}+\sum_{G_{2}} w\left(N\left(v_{2}\right)\right)=0$ which is $0+1$-by + $\sum_{G_{2}} w\left(N\left(v_{2}\right)\right)=0$ therefore $1-$ by $-\mathrm{y}=0$ thus $1=(1+\mathrm{b}) \mathrm{y}$ where is any variable, this is not possible.

All cases are discussed and prove is complete.

We conclude that vertex identi_cation of two - 1 - core vertices cannot give a -1-core vertex. One can answer? does there exist graphs that satisfy the above 6 cases, the answer is illustrated by the next table.

Table 1. Illustration of cases of Theorem 4.3

\begin{tabular}{||c|c|c|c|c|c|c|c||}
\hline \hline Case & $\mathrm{G}_{1}$ & $\mathrm{X}_{1}$ & $\mathrm{G}_{2}$ & $\mathrm{Y}_{1}$ & $\mathrm{G}$ & $\mathrm{Z}_{1}$ & $m_{1}\left(G_{1} \bullet G_{2}\right)$ \\
\hline \hline \hline 1 & $\mathrm{P}_{3}$ & 0 & $\mathrm{P}_{4}$ & 0 & $\mathrm{P}_{6}$ & 0 & 0 \\
& $\mathrm{P}_{3}$ & 0 & $\mathrm{P}_{3}$ & 0 & $\mathrm{P}_{5}$ & $\mathrm{z}$ & +1 \\
\hline 2 & $K_{1,3}$ & 0 & $\mathrm{P}_{3}$ & 0 & $K_{1,3} \bullet P_{3}$ & $\mathrm{z}$ & +1 \\
& $v_{1}$ has \\
& degree 1 & & $\begin{array}{c}v_{2} \text { has } \\
\text { degree 2 }\end{array}$ & & & & \\
\hline 3 & $\mathrm{P}_{2}$ & $\mathrm{x}$ & $\mathrm{P}_{3}$ & 0 & $\mathrm{P}_{4}$ & 0 & -1 \\
\hline 4 & $\mathrm{P}_{2}$ & $\mathrm{x}$ & $\mathrm{P}_{4}$ & 0 & $\mathrm{P}_{5}$ & $\mathrm{z}$ & 0 \\
\hline 5 & $\mathrm{C}_{3}$ & $\mathrm{x}, \mathrm{u}$ & $\mathrm{C}_{3}$ & $\mathrm{y}, \mathrm{v}$ & $C_{3} \bullet C_{3}$ & $\mathrm{x}, \mathrm{y}$ & -2 \\
\hline 6 & $\mathrm{P}_{2}$ & $\mathrm{x}$ & $\mathrm{P}_{2}$ & $\mathrm{y}$ & $\mathrm{P}_{3}$ & $\mathrm{z}=\mathrm{ax}+\mathrm{by}=0$ & -2 \\
\hline \hline
\end{tabular}


Theorem 4.4 For any tree Thaving at least 2 vertices, whose root is an end vertex $v$ then,

$$
m_{-1}\left(C_{3} \bullet T\right) \geq m_{-1}(T)
$$

strictly holds where $T=P_{4 n}$, and equality holds where $T=P_{2}$ Proof. Label the vertices of $\mathrm{C}_{3}$ as $\mathrm{u}_{1}$, $\mathrm{u}_{2}$, and $\mathrm{u}_{3}=\mathrm{v}_{1}$ ( the root vertex) then,in any hczsw of $\mathrm{C}_{3} \bullet \mathrm{T}$ put $\mathrm{w}\left(\mathrm{u}_{1}\right)=\mathrm{x}$ and $\mathrm{w}\left(\mathrm{u}_{2}\right)$ $=-\mathrm{x}$. If $\mathrm{w}\left(\mathrm{v}_{1}\right)=0$ then it is clear that when we remove $\mathrm{u}_{1}$ and $\mathrm{u}_{2}$ from the composite graph $\mathrm{C}_{3} \bullet \mathrm{T}$ the variable $\mathrm{x}$ is removed so the number of independent variables in the hczsw is reduced by 1 . If $\mathrm{w}\left(\mathrm{u}_{3}\right)=\mathrm{y}$, in the hczsw of $\mathrm{T}$, then put $\mathrm{w}\left(\mathrm{u}_{1}\right)$ $=\mathrm{x}$ and $\mathrm{w}(\mathrm{u} 2)=-\mathrm{x}-\mathrm{y}$. Again removing $\mathrm{u}_{1}$ and $\mathrm{u}_{2}$ from the composite graph $\mathrm{C}_{3} \bullet T$ only the variable $\mathrm{x}$ is removed so the number of independent variables in the hczsw is again reduced by 1 . Finally, -1 is not an eigenvalue for $\mathrm{P} 4 \mathrm{n}$, but a hczsw for $\mathrm{C}_{3} \bullet \mathrm{P}_{4 \mathrm{n}}$ that uses 2 variables, hence $\mathrm{m}_{-1}\left(\mathrm{C}_{3} \bullet \mathrm{P}_{4 \mathrm{n}}\right)$ $=\mathrm{m}_{-1}\left(\mathrm{P}_{4 \mathrm{n}}\right)+2$ Also it is clear that $\mathrm{m}_{-1}\left(\mathrm{C}_{3} \bullet \mathrm{P}_{2}\right)=\mathrm{m}_{-1}\left(\mathrm{P}_{2}\right)=1$.

Theorem 4.5 For any connected graph $G$ having at least 2 vertices, with rooted vertex $v$, then

$m_{-1}\left(P_{4 n} \bullet G\right)=m_{-1}(G)$

where the root vertex of $P_{4 n}$ is one of its end vertices.

Proof. Label the vertices of $\mathrm{P}_{4 \mathrm{n}}$ as $\mathrm{u}_{1,1}, \mathrm{u}_{1,2}, \mathrm{u}_{1,3}, \mathrm{u}_{1,4}, \mathrm{u}_{2,1}, \mathrm{u}_{2,2}$, $\mathrm{u}_{2,3}, \mathrm{u}_{2,4}, \ldots, \mathrm{u}_{\mathrm{n}, 1}, \mathrm{u}_{\mathrm{n}, 2}, \mathrm{u}_{\mathrm{n}, 3}, \mathrm{u}_{\mathrm{n}, 4}=\mathrm{v}$ ( the root vertex) then, in any hczsw of $\mathrm{P}_{4 \mathrm{n}} \bullet \mathrm{G}$, if $\mathrm{w}\left(\mathrm{u}_{\mathrm{n}, 4}\right)=\mathrm{x}$, the vertices of $\mathrm{P}_{4 \mathrm{n}}$ will be weighted as $\mathrm{x},-\mathrm{x}, 0, \mathrm{x},-\mathrm{x}, 0, \mathrm{x},-\mathrm{x}, 0, \mathrm{x}$ so, it is clear that when we remove $\mathrm{u}_{1,1}, \mathrm{u}_{1,2}, \mathrm{u}_{1,3}, \mathrm{u}_{1,4}, \mathrm{u}_{2,1}, \mathrm{u}_{2,2}, \mathrm{u}_{2,3}, \mathrm{u}_{2,4}, \ldots, \mathrm{u}_{\mathrm{n}, 1}, \mathrm{u}_{\mathrm{n}, 2}$, $\mathrm{u}_{\mathrm{n}, 3}$ from the composite graph $\mathrm{P}_{4 \mathrm{n}} \bullet \mathrm{G}$ the variable $\mathrm{x}($ or $-\mathrm{x})$ is not removed so the number of independent variables in the hczsw remains the same. If $w\left(u_{n, 4}\right)=0$, in the hczsw of $\mathrm{G}$, the vertices

of $\mathrm{P} 4 \mathrm{n}$ will be weighted as $0,0, \ldots, 0$, Again removing the above vertices from the composite graph $\mathrm{P}_{4 \mathrm{n}} \bullet \mathrm{G}$ no variable will be removed or increased so the number of independent variables in the hczsw again remains the same.

As a usual question, one can ask: given any number $\mathrm{k}$ does there exist a graph or a tree or under a limited diameter for which the null spaces dimension of of -1 is $\mathrm{k}$. So, the following cases are easily constructed:

1) The complete graph with order $n=k+1$ is such a graph of diameter 1 .

2) The vertex identification of the central vertices of $k$ copies of $\mathrm{P}_{5}$ is such a graph ( tree with diameter 4).

3) The vertex identification of the central vertices of $k$ copies $P_{5+6 t,} t=1,2,3, \ldots$ is such a graph ( tree with diameter $4+6 \mathrm{t}$ ).

4) The vertex identification of the (third) vertices of $k$ copies of $\mathrm{P}_{8}$ is such a graph ( tree with diameter 10). 5) The vertex identification of the (third) vertices of $k$ copies $P_{8+6 t}, t=1,2,3, \ldots$ is such a graph ( tree with diameter $12 t+10$ ).

A Fan graph $\mathrm{F}$ with $\mathrm{n}$ arms is de ned to be the graph obtained from identifying a vertex of $n$ copies of $C_{3}$, then $|F|=2 n+1$ with dimension of -1 to be $n$, not a tree, connected graph with minimum diameter 2 . Also, consider the generalized Fan graph $\mathrm{F}^{*}$, with $\mathrm{a}, \mathrm{b}$ and $\mathrm{c}$ arms (Where at least non two of them are zero), which is also a connected graph with diameter 3 and $\mathrm{m}_{-1}\left(\mathrm{~F}^{*}\right)=\mathrm{a}+\mathrm{b}+\mathrm{c}$.

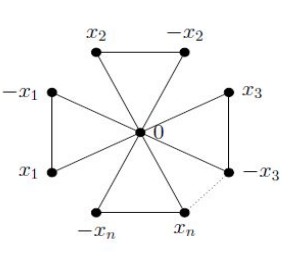

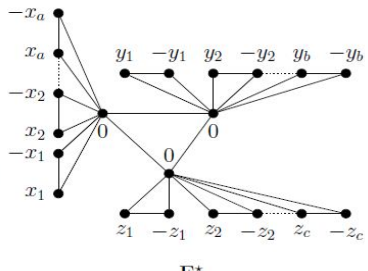

Figure 5. hczsw of the Fan graph $\mathrm{F}$ and generalized Fan graphs $\mathrm{F}^{*}$

\section{NULL SPACES DIMENSION OF -1 IN THE CORONA PRODUCT OF TWO GRAPHS}

In this section, we determine the dimension of the null space of -1 as an eigenvalue in the corona product of two graphs.

Proposition 5.1 For paths $P_{n}, n>0$ and $P_{t}, t>0$ we have: $m_{-1}\left(P_{t} \mathrm{O} P_{n}\right)=\left\{\begin{array}{ccc}t+1 & \text { if } & n=2 \bmod 3 \text { and } t \text { is odd } \\ t & \text { if } & n=2 \bmod 3 \text { and } t \text { is even } \\ 1 & \text { if } & n \neq 2 \bmod 3 \text { and } t \text { is odd } \\ 0 & \text { if } & n \neq 2 \bmod 3 \text { and } t \text { is even }\end{array}\right.$

Proof. Label the vertices of the graph $\mathrm{P}_{\mathrm{t}} \mathrm{OP}_{\mathrm{n}}$ as $\mathrm{v}_{1,1}, \mathrm{v}_{1,2}, \ldots$,

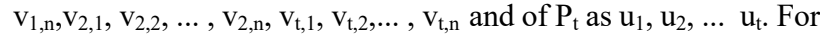
$\mathrm{n}=2 \bmod 3$ and $\mathrm{t}$ is odd the vertices of the ith copy of $\mathrm{P}_{\mathrm{n}}$ are weighted $\mathrm{x}_{\mathrm{ji}},-\mathrm{x}_{\mathrm{ji}}-\mathrm{y}, 0, \mathrm{x}_{\mathrm{ji}},-\mathrm{x}_{\mathrm{ji}}-\mathrm{y}, 0, \ldots, \mathrm{x}_{\mathrm{ji}},-\mathrm{x}_{\mathrm{ji}}-\mathrm{y}$ where $\mathrm{w}\left(\mathrm{u}_{\mathrm{j}}\right)$ $=y$, while the vertices of $P_{n}$ are labeled $y, 0,-y, 0, \ldots,+y$ or $-y$. Hence, there exist $t+1$ independent variables in a hczsw of $\mathrm{P}_{t} O \mathrm{P}_{n}$.

If $t$ is even then, $y=0$ and the number of independent variables is reduced to $t$. For $n \neq 2 \bmod 3$ and $t$ is odd each $x_{i}=0$ and the number of independent variables is reduced to 1 , while if $\mathrm{n} \neq 2$ mod 3 and $t$ is even each $x_{i}=0$ and $y=0$, then the number of independent variables is reduced to 0 .

Proposition 5.2 For any cycle $C_{n}$ and path $P_{t}$ then:
$m_{-1}\left(P_{t} O C_{n}\right)=\left\{\begin{array}{cl}2 t+1 \text { if } \quad n=3 \text { and } t \text { is odd } \\ 2 t \quad \text { if } \quad n=3 \text { and } t \text { is even } \\ t \quad \text { if } n>3 \text { and } n=0 \text { mod } 3 \\ 0 \quad \text { otherwise }\end{array}\right.$

Proof. Label the vertices of the graph $\mathrm{P}_{\mathrm{t}} \mathrm{OC}_{\mathrm{n}}$ as $\mathrm{v}_{1,1}, \mathrm{v}_{1,2}, \ldots, \mathrm{v}_{1, \mathrm{n}}$, $\mathrm{v}_{2,1}, \mathrm{v}_{2,2}, \ldots, \mathrm{v}_{2, \mathrm{n}}, \ldots, \mathrm{v}_{\mathrm{t}, 1}, \mathrm{v}_{\mathrm{t}, 2}, \ldots, \mathrm{v}_{\mathrm{t}, \mathrm{n}}$ and of $\mathrm{P}_{\mathrm{t}}$ as $\mathrm{u}_{1}, \mathrm{u}_{2}, \ldots, \mathrm{u}_{\mathrm{t}}$. For $\mathrm{n}=3$ and $\mathrm{t}$ is odd the vertex weighting of $\mathrm{P}_{\mathrm{t}} \mathrm{OC}_{3}$ is $\mathrm{x}_{1,1}, \mathrm{x}_{2,1}$, $\mathrm{x}_{1,1}-\mathrm{x}_{2,1}-\mathrm{y}, \mathrm{x}_{1,2}, \mathrm{x}_{2,2},-\mathrm{x}_{1,2}-\mathrm{x}_{2,2}, \mathrm{x}_{1,3}, \mathrm{x}_{2,3},-\mathrm{x}_{1,3}-\mathrm{x}_{2,3}+\mathrm{y}, \ldots, \mathrm{x}_{1, \mathrm{i}}, \mathrm{x}_{2, \mathrm{i}}$, $-\mathrm{x}_{1, \mathrm{i}}-\mathrm{x}_{2, \mathrm{i}}-\mathrm{y}($ or $+\mathrm{y}), \ldots, \mathrm{x}_{1, \mathrm{t}}, \mathrm{x}_{2, \mathrm{t}},-\mathrm{x}_{1, \mathrm{t}}-\mathrm{x}_{2, \mathrm{t}}-\mathrm{y}($ or $+\mathrm{y})$, while the vertices of $\mathrm{P}_{\mathrm{n}}$ are weighted by, $\mathrm{y}, 0, \mathrm{y}, 0,:::,+\mathrm{y}($ or $\mathrm{y})$. That is the vertex uj is weighted by, $y$ if, $i=1 \bmod 3$ or 0 if $i=2 \bmod$ 3 or $-y$ if $i=0 \bmod 3$, respectively. Hence, there exist $2 t+1$ independent variables in a hczsw of $\mathrm{P}_{t} \mathrm{OC}_{3}$.

If $\mathrm{n}=3$ and $\mathrm{t}$ is even then the variable $\mathrm{y}$ is vanished. If $\mathrm{n}>3$ and $\mathrm{n}=0 \bmod 3$ then $\mathrm{y}$ will vanish and $\mathrm{x}_{2, \mathrm{i}}=-\mathrm{x}_{1, \mathrm{i}}$ so another $\mathrm{t}$ variables will vanish. If $\mathrm{n} \neq 0$ mod 3 then there exist no non trivial hczsw for $\mathrm{P}_{t} \mathrm{OC}_{\mathrm{n}}$ and in this case $\mathrm{m}_{-1}\left(\mathrm{P}_{t} \mathrm{OC}_{\mathrm{n}}\right)=0$.

Proposition 5.3 For any complete graph $K_{n}$ : $m_{-1}\left(P_{t} O K_{n}\right)=\left\{\begin{array}{cll}(n-1) t+1 & \text { if } & t \text { is odd } \\ (n-1) t & \text { if } & t \text { is even }\end{array}\right.$ Proof. It is an extension of the prove of the first part of Proposition 5.1.

\section{CONSTRUCTING -1-NUT GRAPHS}

In this section, two classes of -1-nut graphs are constructed in the manner of nut graphs defined by (Sciriha, 2007)

Definition 6.1 A graph $\mathrm{G}$ is said to be a -1 - nut graph if -1 is an eigenvalue of $G$ with multiplicity 1 such that every vertex of $G$ has a non zero weight in its hczsw.

Thus, if $\mathrm{G}$ is a -1 - nut graph then there exist only one core vector inthe null space of the matrix $A(G)+I$ whose size is $n$.

Proposition 6.2 The graph $\mathrm{C}_{n} O K_{3}^{c}$ is a-1-nut graph for each $n$, $n>2$.

Proof. For each $\mathrm{n}$ the graph $\mathrm{C}_{\mathrm{n}} \mathrm{OK}_{3}^{\mathrm{c}}$ is a semi-regular graph with degree set $\{1,5\}$ and order $4 \mathrm{n}$. There is only a unique hczsw for this graph which uses only one variable say $\mathrm{x}$, where $\mathrm{x}$ is the weight of each vertex of degree 5 and $-\mathrm{x}$ is the weight of each vertex of degree one. Thus, the graph $\mathrm{C}_{n} \mathrm{OK}_{3}^{\mathrm{c}}$ is a $\quad-1$ nut graph. 
Proposition 6.3 The graph $C_{n} O K_{n}^{c}$ is a-1-nut graph for each $n, n>0$.

Proof. For each $n$ the graph $\mathrm{C}_{\mathrm{n}} \mathrm{OK}_{3}^{\mathrm{c}}$ is a semi-regular graph with degree set $\{1,2 \mathrm{n}-1\}$ and order $\mathrm{n}^{2}+\mathrm{n}$. There is only a unique hczsw for this graph which uses only one variable say $\mathrm{x}$, where $\mathrm{x}$ is the weight of each vertex of degree $2 n-1$ and $\mathrm{x}$ is the weight of each vertex of degree one. Thus, the graph $\mathrm{C}_{\mathrm{n}} \mathrm{O} \mathrm{K} \mathrm{K}_{3}^{\mathrm{c}}$ is a-1-nutgraph.
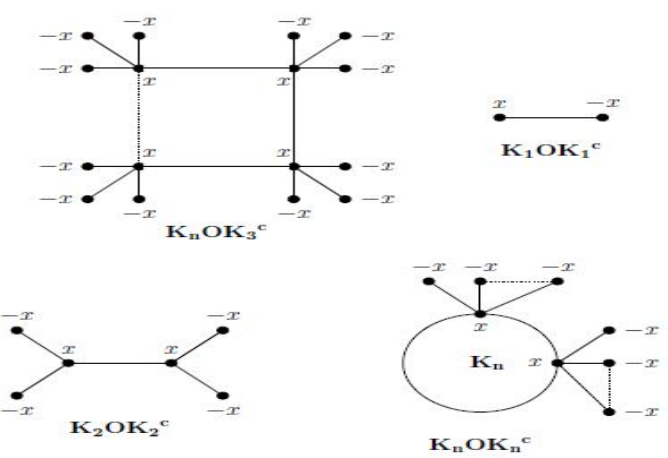

Figure 5. Two classes of -1 - nut graphs.

So, the order of the smallest nut graph is 2 and out of non trees -1-nut graphs, the minimum order is 12 .

Moreover, the only -1-nut graphs which are tree are namely $\mathrm{P}_{2}$ and $\mathrm{K}_{1,3} \mathrm{O} \mathrm{P}_{3}$ mentioned in the above table.
Also it is clear that, the vertex identification of any two -1 - nut graphs is not a $-1-$ nut graph.

\section{REFERENCES}

Ali , D.A., Gauci, J.B., Sciriha, I., \& Sharaf, K.R. (2016a). Coalescing Fiedler and Core Vertices. Czechoslovak Mathematical 971985.

Ali , D.A., Gauci, J.B., Sciriha, I., \& Sharaf, K.R. (2016b). Nullity of a Graph with a Cut-edge. Communication in Mathematical and in Computer Chemistry, 76, 771-791.

Ali, D.A., Gauci, J.B., Sciriha, I., \& Sharaf, K.R. (2019). The Conductivity of Superimposed Key-graphs with a Common One Dimensional Adjacency Nullspace. ARS Mathematica Contemporanea, 16, 1-15.

Brouwer, A. E., \& Haemers, W.H. (2011). Spectra of Graphs. Springer. Cheng, B., \& Liu, B. (2007). On the Nullity of graphs. Journal of Linear Algebra, 16 .

Cvetkovic, D. M., Doob, M., \& Sachs, H. (New York 1979). Spectra of Graphs-Theory and Applications. Acamemic Press.

Mohan, S., Geetha, J., \& Somasundaram,KB. (2017). Total Coloring of the Corona Product of two Graphs. Australasian Journal of Combinatorics, 68 .

Mohiaddin, G.H., \& Sharaf, K.R. (2018). Construction and Nullity of Some Classes of Smith Graphs. IEEE Xplore.

Sciriha, I. (2007). A characterization of singular graphs. Electronic Journal of Linear Algebra, 16, 451-462.

Sharaf, K.R., \& Rashed, P.A. (2002). On the Degree of the Singular of a Graph. J. of Duhok University, (5), 133-138. 